See Article page XXX.

\section{Commentary: Redo heart transplantation: Lessons learned over the past 20 years}

\section{Valluvan Jeevanandam, MD}

Redo heart transplantation (HT) is controversial. Previous studies have shown a lower survival rate and higher complications compared with primary HT. Hess and colleagues ${ }^{1}$ reviewed the United Nations Organ Sharing database over a 20 -year period to readdress this topic. They similarly conclude that redo HT has worse short- and long-term outcomes even after propensity matching for pretransplant risk factors.

Redo HT is performed for 3 main groups of patients: those with primary graft dysfunction (PGD), acute rejection, or for chronic rejection manifested as allograft dysfunction or transplant arteriopathy. Results after redo HT are dependent on the immunological status of the patient as well as pretransplant risk factors. Patients with PGD make up about $13 \%$ of redo HT patients. The primary therapy for PGD is prolonged circulatory support to allow time for the heart to recover. If the grafts do not recover, there are not many any other options for survival other than redo HT. Because PGD is primarily due to preservation and reperfusion injury, recipient immunological status is of less concern. These patients not only have undergone a long cardiopulmonary bypass period, but also usually have prolonged support with extracorporeal membrane oxygenation, are ventilated, and have end organ dysfunction. Because their need for treatment is urgent, the tendency is to use more marginal organs. As demonstrated by the authors, these patients have the worst short- and long-term outcomes.

\footnotetext{
From the Section of Cardiac Surgery, Department of Surgery, University of Chicago Medicine, Chicago, Ill.

Disclosures: The author reported no conflicts of interest.

The Journal policy requires editors and reviewers to disclose conflicts of interest and to decline handling or reviewing manuscripts for which they may have a conflict of interest. The editors and reviewers of this article have no conflicts of interest.

Received for publication Sept 29, 2021; revisions received Sept 29, 2021; accepted for publication Sept 30, 2021.

Address for reprints: Valluvan Jeevanandam, MD, Section of Cardiac Surgery, Department of Surgery, University of Chicago Medicine, 5841 S Maryland Ave, MC5040, Chicago, IL 60637 (E-mail: jeevan@uchicago.edu).

J Thorac Cardiovasc Surg 2021; $\mathbf{\square}: 1-2$

$0022-5223 / \$ 36.00$

Copyright (c) 2021 by The American Association for Thoracic Surgery

https://doi.org/10.1016/j.jtcvs.2021.09.054
}

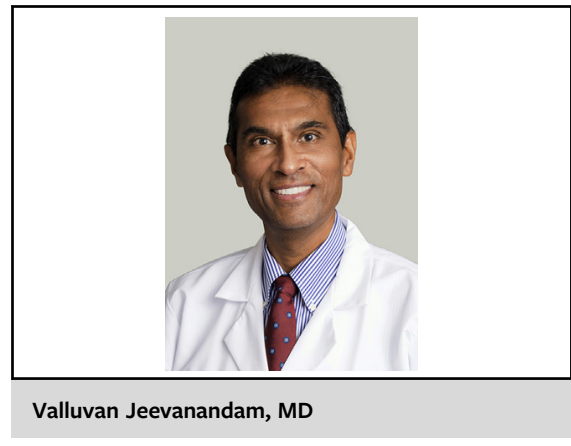

CENTRAL MESSAGE

Redo heart transplantation is associated with poorer shortand long-term outcomes than primary heart transplantation. Proper patient selection might mitigate the risks.

Redo HT for acute rejection makes up about $4 \%$ of patients. Redo HT outcomes in this subgroup are driven mostly by the immunological response. Acute rejection can occur within the first year or later in a patient's course. For patients who are hemodynamically compromised and require mechanical support, results will be influenced by the number of preoperative risk factors such as renal, pulmonary, hepatic, and neurologic dysfunction.

Redo HT for chronic rejection or transplant arteriopathy has the best outcomes. These patients are usually clinically stable and quiescent from an immunological viewpoint. Immunological injury to the fresh allograft is of less concern than for patients with acute rejection. These patients might also undergo transplant before experiencing end organ dysfunction and before requiring mechanical and respiratory support. These patients also have lessinflammatory chest cavities for reoperation surgery. Many of these patients will have abnormal renal function due to long-term calcineurin inhibitors. Some might require a combined heart-kidney transplant. A glomerular filtration rate of at least $40 \mathrm{~mL} / \mathrm{min} / 1.73 \mathrm{~m}^{2}$ should suffice in these patients because the kidneys have already been subjected to and have accommodated nephrotoxic agents.

Redo HT is associated with poorer short- and long-term outcomes than primary HT. Proper patient selection might mitigate the risks because there are 3 distinct subgroups of patients each with their own immunologic and preoperative risk factors and distinct outcomes. Because long-term 
left ventricular assist device therapy is generally not possible, redo HT is the only option for this small but high-acuity patient population.

\section{Reference}

1. Hess NR, Hickey GW, Sultan I, Kilic A. Redo orthotopic heart transplantation in the current era. J Thorac Cardiovasc Surg. 2021. XX:XX-X. 